\title{
The isoflavone metabolite 6-methoxyequol inhibits angiogenesis and suppresses tumor growth
}

Sofia Bellou ${ }^{1,2}$, Evdoxia Karali2, ${ }^{2,6}$, Eleni Bagli², Nawaf Al-Maharik ${ }^{3}$, Lucia Morbidelli ${ }^{4}$, Marina Ziche ${ }^{4}$, Herman Adlercreutz ${ }^{5}$, Carol Murphy ${ }^{2}$ and Theodore Fotsis ${ }^{2,6^{*}}$

\begin{abstract}
Background: Increased consumption of plant-based diets has been linked to the presence of certain phytochemicals, including polyphenols such as flavonoids. Several of these compounds exert their protective effect via inhibition of tumor angiogenesis. Identification of additional phytochemicals with potential antiangiogenic activity is important not only for understanding the mechanism of the preventive effect, but also for developing novel therapeutic interventions.

Results: In an attempt to identify phytochemicals contributing to the well-documented preventive effect of plant-based diets on cancer incidence and mortality, we have screened a set of hitherto untested phytoestrogen metabolites concerning their anti-angiogenic effect, using endothelial cell proliferation as an end point. Here, we show that a novel phytoestrogen, 6-methoxyequol (6-ME), inhibited VEGF-induced proliferation of human umbilical vein endothelial cells (HUVE) cells, whereas VEGF-induced migration and survival of HUVE cells remained unaffected. In addition, 6-ME inhibited FGF-2-induced proliferation of bovine brain capillary endothelial (BBCE) cells. In line with its role in cell proliferation, 6-ME inhibited VEGF-induced phosphorylation of ERK1/2 MAPK, the key cascade responsible for VEGF-induced proliferation of endothelial cells. In this context, 6-ME inhibited in a dose dependent manner the phosphorylation of MEK1/2, the only known upstream activator of ERK1/2. 6-ME did not alter VEGFinduced phosphorylation of p38 MAPK or AKT, compatible with the lack of effect on VEGF-induced migration and survival of endothelial cells. Peri-tumor injection of 6-ME in A-431 xenograft tumors resulted in reduced tumor growth with suppressed neovasularization compared to vehicle controls $(P<0.01)$.
\end{abstract}

Conclusions: 6-ME inhibits VEGF- and FGF2-induced proliferation of ECs by targeting the phosphorylation of MEK1/ 2 and it downstream substrate ERK1/2, both key components of the mitogenic MAPK pathway. Injection of 6-ME in mouse A-431 xenograft tumors results to tumors with decreased neovascularization and reduced tumor volume suggesting that 6-ME may be developed to a novel anti-angiogenic agent in cancer treatment.

Keywords: Angiogenesis, VEGF, Phytoestrogen, MAPK, A-431 xenograft

\footnotetext{
*Correspondence: thfotsis@uoi.gr

${ }^{2}$ Department of Biomedical Research, Foundation of Research and

Technology-Hellas, Institute of Molecular Biology \& Biotechnology, University

Campus, 45110, loannina, Greece

${ }^{6}$ Laboratory of Biological Chemistry, Medical School, University of loannina,

45110, Ioannina, Greece

Full list of author information is available at the end of the article
}

\section{Biomed Central}

(c) 2012 Bellou et al.; licensee BioMed Central Ltd. This is an Open Access article distributed under the terms of the Creative Commons Attribution License (http://creativecommons.org/licenses/by/2.0), which permits unrestricted use, distribution, and reproduction in any medium, provided the original work is properly cited. 


\section{Introduction}

Physiological angiogenesis is a strictly regulated finetuned process. The local balance between inducers and inhibitors of angiogenesis is critical in determining the generation or not of new vessels. Whenever this balance is perturbed pathological, uncontrolled, excessive angiogenesis occurs. Psoriasis, rheumatic arthritis and diabetic retinopathy constitute some of the diseases in which pathological angiogenesis contributes to their pathogenesis. However, tumor angiogenesis is the most striking manifestation of abnormal angiogenesis. Indeed, it has been demonstrated that formation of new blood vessels is required for tumor growth beyond a diameter of 1-2 $\mathrm{mm}$.

Vascular endothelial growth factor A (VEGFA), also referred to as VEGF, represents a critical inducer of tumor angiogenesis and is the first-choice target of antiangiogenic therapies tested in clinical trials [1]. VEGF belongs to a subfamily of secreted, dimeric glycoproteins of approximately $40 \mathrm{kDa}$, which in turn belongs to the platelet-derived growth factor (PDGF) superfamily. In mammals, VEGF family consists of VEGF-A, B, C, D and placental growth factor 1 and 2 (PlGF1 and 2). Specifically VEGF exists as multiple isoforms, resulting from alternative splicing. The most predominant isoform is VEGF165 (a 165-amino acid protein), which is overexpressed in a variety of human solid tumors [2,3]. All VEGF molecules/ligands transduce their signal through their binding to VEGF receptor $-1,-2$ and -3 . However, VEGFR-2 is the key molecule for VEGF signaling in the tumor micro-environment including vascular permeability and endothelial cell proliferation[2,4]. Several cascades emanating from the VEGF/VEGFR2 complex regulate critical angiogenic responses of endothelial cells. Endothelial cell proliferation is regulated by activation of PLC $\gamma$, a SH2-domain-containing molecule that interacts directly with activated VEGFR-2 and mediates the phosphorylation of mitogen-activated protein kinase (MAPK)/ extracellular - signal-regulated kinase 1/2 (ERK1/2) cascade [5]. VEGF enhances survival of endothelial cells using the PI3K/AKT pathway, whereas it stimulates endothelial cell migration through $\mathrm{p} 38$ MAPK phosphorylation [6]. Signaling cascades of the VEGF/VEGFR2 complex result in the expression of dual specificity phosphatases (DUSP) 1 \& 5, which dephosphorylate and inactivate MAPKs, functioning as an auto-regulatory circuit [7].

Consumption of plant-derived diets exerts a preventive effect on cancer incidence in humans. Several dietary phytochemicals exhibit anti-mitotic and/or anti-angiogenic activity mediating the protective effect of vegetarian diets on cancer. In this context, we have demonstrated that the isoflavonoid genistein is a potent inhibitor of tumor cell proliferation and angiogenesis [8]. Subsequently, we have shown that several of the isomeric flavonoids exhibited similar anti-angiogenic activity as genistein [9]. In particular, luteolin inhibited VEGF-induced angiogenesis by targeting VEGF/VEGFR2-induced PI3K activity. Detailed elucidation of the mechanism demonstrated that luteolin compromised VEGF-induced survival of HUVECs via blockage of PI3K/Akt-dependent pathways, whereas inhibition of the PI3K/p70 S6K pathway mediated the anti-mitotic effects of the compound on HUVECS [10]. In the present study, we have screened additional isoflavonoids for anti-angiogenic activity and identified that 6-methoxyequol inhibits VEGF-induced MEK1/2 phosphorylation and endothelial cell proliferation leaving unaffected the migratory and survival functions of VEGF. Treatment of xenograft A-431 tumors in mice using oral administration of 6-ME failed to reduce the volumes of the tumors, because the compound failed to achieve sufficient plasma levels as documented using an HPLCCEAD method. However, injecting directly 6-ME to the xenograft tumors, to bypass the low bioavailability, resulting in a statistically significant reduction of tumor volume compared to controls and suppressed vascularization.

\section{Materials and methods \\ Antibodies and chemicals}

Human VEGF165 was purchased from ImmunoTools (ImmunoTools $\mathrm{GmbH}$, Friesoythe, Germany). Rabbit polyclonal anti-phospho-p38, anti-ERK1/2, anti-phosphoERK1/2, anti-phospho-Akt and anti-Akt antibodies were obtained from Cell Signaling (Cell Signaling Technology, Inc, Beverley, MA). Anti-BrdU was from Sigma (Sigma, St. Louis, MO). All secondary antibodies were purchased from Jackson ImmunoResearch Europe Ltd, UK. CycleTEST PLUS DNA Reagent kit was from Becton Dickinson Biosciences.

\section{Cell culture}

Human endothelial cells from umbilical vein (HUVEC) were plated on dishes pre-coated with rat collagen type I (Becton Dickinson Biosciences) and cultured in M199 medium supplemented with $20 \%$ fetal calf serum (FCS), 50 micrograms $/ \mathrm{ml}$ endothelial cell growth supplement (ECGS, Sigma), heparin $10 \mathrm{u} / \mu \mathrm{l}$ (Sigma) and $1 \%$ penicillin-streptomycin. All media and sera for cell culture were purchased from Invitrogen and were endotoxinfree. 6-methoxyequol was tested for endotoxin content using the QCL1000 kit from BioWhittaker, Inc. For all experiments 6-methoxyequol was resuspended in DMSO/ ethanol, $1 / 1$ by volume, and added directly to the culture medium. Cells not receiving 6-methoxyequol were incubated in the corresponding volume of DMSO/ethanol.

\section{Primary cell growth assay}

Primary bovine brain capillary endothelial (BBCE) cells were split into 12-well dishes at 5,000 cells per well and 24 h later cell stimulated with FGF2 $(2.5 \mathrm{ng} / \mathrm{ml})$ in the 
absence or presence of 6-methoxyequol at various concentrations. After 2 days, cells were again stimulated or not by FGF2 in the absence or presence of 6-methoxyequol and the next day cells were counted.

\section{Cancer cell growth assay}

Hela, LnCAP, T24 (Human bladder carcinoma) or MCF7 (Human breast adenocarcinoma) cancer cells were split into 12-well plates either at 5,000, in case of Hela, T24 and MCF7 or at 20,000 in case of LnCAP, cells per well and $24 \mathrm{~h}$ later cells were treated or not with various concentrations of 6-methoxyequol. After 2 days, cells were again treated or not with 6- methoxyequol and the next day cells were counted.

\section{Apoptosis assay}

For analysis by flow cytometry, HUVECs were serum starved for $6 \mathrm{~h}$ in medium containing 5\% FCS and treated with VEGF (50 ng/ml) for $18 \mathrm{~h}$ in the presence or absence of 6-methoxyequol $(10 \mu \mathrm{M})$ for the same period of time. At the end of the incubation time, floating and adherent cells were collected in ice-cold PBS, stained with propidium iodine using the CycleTEST PLUS DNA Reagent kit and processed for flow cytometric analysis using a Becton Dickinson Fluorescence Activated Cell Scanner (FACS). The percentage of cells with sub-G1 DNA content was considered as the cell population that had undergone apoptosis.

\section{Proliferation assay (BrdU incorporation)}

HUVECs were grown on collagen-coated coverslips and serum starved in medium containing 5\% FCS, $1 \%$ pen/ strep and heparin for $18 \mathrm{~h}$. Cells were induced with VEGF $(50 \mathrm{ng} / \mathrm{ml}$ ) in the absence or presence of various concentrations of 6-methoxyequol for $24 \mathrm{~h}$. Bromodeoxyuridine (BrdU; Sigma, St. Louis, MO) was added $6 \mathrm{~h}$ before the VEGF-induction was complete. Cells were fixed in $3.7 \%$ paraformaldehyde, quenched with $50 \mathrm{mM}$ ammonium chloride for $15 \mathrm{~min}$, permeabilized with $0.1 \%$ Triton X-100 for $4 \mathrm{~min}$, and non-specific sites were blocked with fetal serum. The proliferating cells were detected with an anti-BrdU antibody. Coverslips were mounted in Mowiol and viewed using Leica DM IBRE microscope.

\section{Cell migration assay}

Confluent HUVE cell monolayers were wounded with a sterile plastic pipette tip, cultured in M199 medium supplemented with 5\% FCS and induced with VEGF (10 ng/ $\mathrm{ml})$ in the presence or absence of 6-methoxyequol $(10 \mu \mathrm{M})$. Cells were placed in a $37^{\circ} \mathrm{C}, 5 \% \mathrm{CO} 2$ chamber and monitored using a Leica DM IBRE microscope equipped with a HRD060-NIK CCD-camera (Diagnostic Instruments, Sterling Heights, MI, USA) and metamorph software. Frames were taken every $10 \mathrm{~min}$ for $16 \mathrm{~h}$. Results were expressed as number of cells per centimetre of wound.

\section{Tube formation assay}

Matrigel was thawed on ice overnight and spread evenly over each well $(500 \mu \mathrm{l})$ of a 24 -well plate. The plates were incubated for $30 \mathrm{~min}$ at $37^{\circ} \mathrm{C}$ to allow the matrigel to polymerize. HUVECs were seeded on coated plated at $4 \times 10^{4}$ cells/well in M199 supplemented with 5\% FCS in the presence or absence of 6-methoxyequol at various concentrations $(1-50 \mu \mathrm{M})$. Plates were incubated for $12 \mathrm{~h}$ at $37^{\circ}$ C. Tube formation was observed using an inverted phase contrast microscope (Zeiss Axiovert S-100; Germany).

\section{Phosphorylation of MAP kinases}

HUVECs were cultured in M199 supplemented with $20 \%$ FCS, ECGS, heparin \& pen/strep until $80 \%$ confluence. Cells were serum starved for $2 \mathrm{~h}$ in medium containing 0\% FCS and then treated with VEGF $(50 \mathrm{ng} / \mathrm{ml}$ ) in the presence or absence of either 6-methoxyequol $(1,5$,or $10 \mu \mathrm{M})$ or DMSO for $15 \mathrm{~min}$. Cells were washed with ice-cold PBS and lysed in lysis buffer (1\% SDS supplemented with protease and phosphatase inhibitors). The lysates were resuspended in Laemmli buffer, subjected to SDS-PAGE and blotted onto a nitrocellulose membrane. Phosphorylated ERK1/2 and p38 were detected using specific rabbit polyclonal antibodies and an anti-rabbit peroxidase-conjugated secondary antibody, followed by detection using a chemiluminescence-based system. The membranes were then stripped and reprobed with antibodies against ERK1/2 and p38 to normalize the phosphorylation data against expression of the kinases.

\section{qRT-PCR experiment}

Quantitative Reverse Transcription-PCR (qRT-PCR) experiments were performed using The LightCycler ${ }^{\circledR} 2.0$ Instrument (Roche Diagnostics GmbH, Mannheim, Germany) and QuantiTect SYBR Green RT-PCR Kit (Qiagen, GmbH, Germany). Total RNA was isolated after 15 and $30 \mathrm{~min}$ treatment with VEGF $(50 \mathrm{ng} / \mathrm{ml})$ in the absence or presence of 6- methoxyequol $(10 \mu \mathrm{M})$.

\section{Synthesis of 6-methoxyequol}

To test 6-ME in animal models considerably larger quantities were required. Since, this compound is not commercially available we undertook its synthesis as described in detail in the Additional file 1. In brief, starting from 6-methoxyresorcinol and 4-hydroxyphenylacetic acid the desired deoxybenzoin was first obtained in $48 \%$ yield. Treatment of the deoxybenzoin with $N, N$ dimethylformamid (DMF) in the presence of methanesulfonyl chloride at $70^{\circ} \mathrm{C}$ generated glycitein, which was hydrogenated using $10 \% \mathrm{Pd} / \mathrm{C}$ to 6-methoxyequol in high yield and purity. A detailed analysis of 1-(2,4dihydroxy-5-methoxyphenyl)-2-(4'-hydroxyphenyl) ethanone, 7,4'-Dihydroxy-6-methoxyisoflavone (Glycitein) and 7,4'-Dihydroxy-6-methoxyisoflavane synthesis is described in. 


\section{In vivo experiments}

To assess the in vivo anti-angiogenic/anti-tumor activity of 6-methoxyequol, female immunodeficient mice (5-8 weekold BALB/c nude mice, Charles River, Milan, Italy), kept with ad libitum water and Protein Rodent Maintenance Diet (Harlan n. 2014), were inoculated subcutaneously in the right flank with $107 \mathrm{~A}-431$ cells in a volume of $50 \mu \mathrm{l}$ (Morbidelli et al., Clinic Cancer Res, 2003; Bagli et. al., Cancer Res, 2004). After 9 days, when tumors reached a volume of $170 \mathrm{~mm}^{3}$, animals were randomly assigned to 2 different experimental groups (9-10 mice per group). Peri-tumor treatment with 6-methoxyequol $(5 \mathrm{\mu g} /$ day/ mice) or vehicle then began. The local peri-tumor treatment was performed at the dose of $5 \mu \mathrm{g} / 50 \mu \mathrm{l} / \mathrm{mouse} /$ day. The vehicle containing the same concentrations of solvents ( $1 \%$ ethanol $+1 \%$ DMSO) was used as control. Daily treatment was performed for 10 consecutive days. Serial caliper measurements of perpendicular diameters were used to calculate tumor volume using the following formula: (shortest diameter $\mathrm{x}$ longest diameter $\mathrm{x}$ thickness of the tumor in $\mathrm{mm}$ ). Data are reported as tumor volume in $\mathrm{mm}^{3}$. Experiments have been performed in accordance with the guidelines of the European Economic Community for animal care and welfare (EEC Law No. 86/609) and National Ethical Committee. Animals were observed daily for signs of cytotoxicity and were sacrificed by $\mathrm{CO}_{2}$ asphyxiation. At day 10 animals were sacrificed and each tumor was immediately frozen in liquid nitrogen. $7 \mu \mathrm{m}$-thick cryostat sections were stained with hematoxylin and eosin and adjacent sections were used for immunohistochemical staining with the anti-ED-B monoclonal antibody after fixation in absolute cold acetone.

In the set of mice treated orally with 6-ME, the compound was firstly dissolved in 50\% ethanol and 50\% DMSO and then diluted with extra pure olive oil (final $0,25 \%$ ethanol and 0,25\% DMSO). We have used as vehicle olive oil with the same amount of solvents. The daily dose of 6-ME was $100 \mathrm{mg} / \mathrm{kg}$ administered by lavage (200 $\mathrm{\mu l} /$ mouse). Treatment started when tumors were palpable and continued until day 11, the day of sacrifice. To accesses 6-ME bioavailability in mice, we determined 6-ME in urine and plasma as described in Additional file 1.

\section{Results}

Screening of flavonoids revealed that 6-methoxyequol is a specific inhibitor of endothelial cell proliferation exhibiting minor anti-mitotic effect on tumor cells

We screened a selection of isoflavonoids on endothelial cell proliferation seeking to identify additional structures with antiangiogenic activity compared to that of genistein. From the 28 isoflavonoids tested, only 6-methoxyequol (6-ME) had a strong inhibitory effect on FGF2-induced endothelial cell (BBCE) proliferation exhibiting an IC50 of approximately $3 \mu \mathrm{M}$ (Table 1 and Figure 1A), slightly
Table 1 IC50 list of isoflavonoids tested on endothelial cell proliferation

\begin{tabular}{|c|c|c|}
\hline & Compound & $\mathrm{IC} 50(\mu \mathrm{M})$ \\
\hline 1 & 3',8-Dinitro-7-hydroxy-4-methoxyisoflavone & $>50$ \\
\hline 2 & 7-Hydroxy-4-methoxy-3',5',8-trinitroisoflavone & 45.9 \\
\hline 3 & 4,7-Dihydroxy-3',5',8-trinitroisoflavone & $>50$ \\
\hline 4 & 3'-Nitro-2,4,4'-trihydroxydeoxybenzoin & $>50$ \\
\hline 5 & 2,4-Dihydroxy-4'-methoxy-5-nitrodeoxybenzion & $>50$ \\
\hline 6 & 5,7-Dihydroxy-4'-nitroisoflavone & 14.1 \\
\hline 7 & 7-Hydroxy-4'-methoxy-8-nitroisoflavone & $>50$ \\
\hline 8 & 3'-Nitro-5,7,4'-trihydroxyisoflavone & 35.2 \\
\hline 9 & 7,4'-Dihydroxy-3'-nitroisoflavone & 42.3 \\
\hline 10 & 5,7-Dihydroxy-2'-methyl-4'-nitroisoflavone & 19.5 \\
\hline 11 & 8,4'-Dinitro-7-hydroxyisoflavone & $>50$ \\
\hline 12 & 4'-Amino-5,7-dihydroxyisoflavone & $>50$ \\
\hline 13 & 8'-Amino-7-hydroxy-4'-methoxyisoflavone & 31.7 \\
\hline 14 & 3'-Amino-5,7,4'-trihydroxyisoflavone & 24.8 \\
\hline 15 & 3'-Amino-7,4'-dihydroxyisoflavone & 37.5 \\
\hline 16 & 4'-Amino-5,7-dihydroxy-2'-methylisoflavone & $>50$ \\
\hline 17 & 4',8-Diamino-7-hydroxyisoflavone & $>50$ \\
\hline 18 & 8,4'-Diamino-7,5,dihydroxyisoflavone & $>50$ \\
\hline 19 & 7,4'-Dihydroxy-8,5'-dinitroisoflavone & 42.2 \\
\hline 20 & 4'-methylequol & 28 \\
\hline 21 & 3'-methoxyequol & 19 \\
\hline 22 & 6-methoxyequol & 3 \\
\hline 23 & 6'-OH-ODMA & 38 \\
\hline 24 & 4'-O-methylequol & 25 \\
\hline 25 & 7'-Hydroxyenterolactone & $>50$ \\
\hline 26 & Pinoresinol & 38 \\
\hline 27 & Luteolin & 5 \\
\hline 28 & Genistein & 5 \\
\hline
\end{tabular}

lower than that of genistein and luteolin (IC50 around $5 \mu \mathrm{M})[9,10]$. The antimitotic effect of 6-methoxyequol appeared to be specific to endothelial cells as 6-ME was devoid of any antimitotic effect on 4 different cancer cell lines at a concentration of $6.25 \mu \mathrm{M}$, even though at higher doses an inhibitory/toxic effect could be observed (Figure 1B). Moreover, 6-ME did not affect proliferation of human fibroblasts even at $20 \mu \mathrm{M}$ concentration (Additional file 1: Figure 1A and B). The inhibitory effect of 6-ME on endothelial cells was consistent as it inhibited also VEGF-induced proliferation of HUVECs (Figure 1C).

\section{6-methoxyequol does not influence VEGF-induced survival of endothelial cells}

To exclude an inhibitory (apoptotic) effect of 6-ME on VEGF-induced survival of endothelial cells, we tested the effect of 6-ME on VEGF-treated endothelial cells following serum starvation. Withdrawal of serum is well known to induce endothelial cell apoptosis, which is 


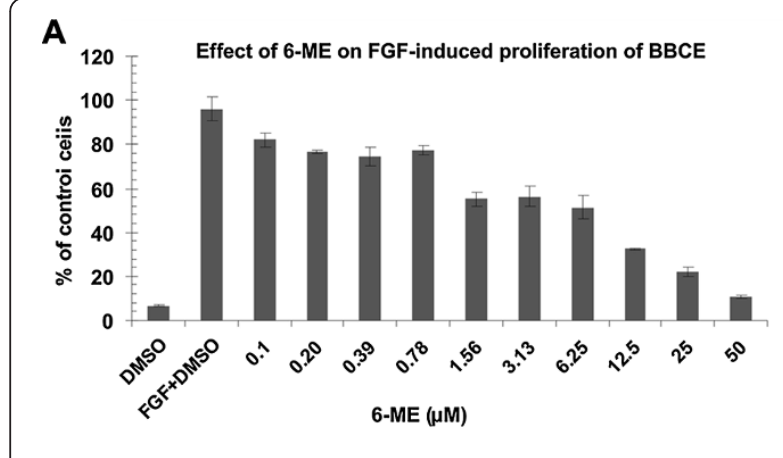

B

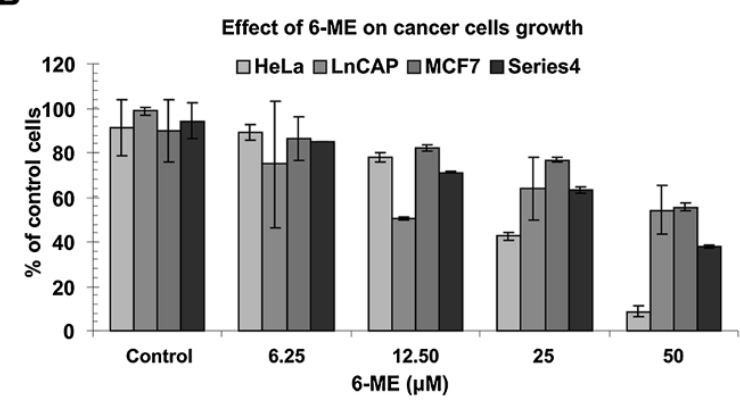

C

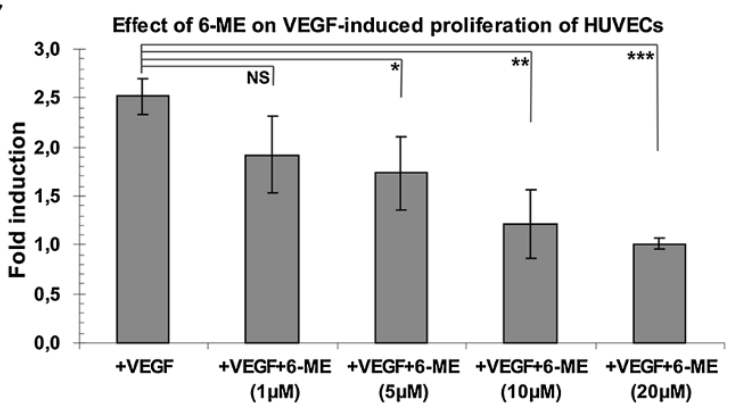

Figure 1 Effect of 6-ME on endothelial and cancer cell proliferation. (A) BBCE cells were seeded $24 \mathrm{~h}$ before stimulation by FGF $(2.5 \mathrm{ng} / \mathrm{ml})$ in the absence or presence of different concentrations of 6-ME. After $48 \mathrm{~h}$ cells were again stimulated with FGF $(2.5 \mathrm{ng} / \mathrm{ml})$ in the absence or presence of 6-ME and next day cells were counted. (B) HeLA, LnCAP, MCF7 or T24 cells were treated or not with various concentrations of 6-ME. After 48 h 6-ME was added again and $24 \mathrm{~h}$ later cells were counted. (C) HUVE cells were serum starved for $6 \mathrm{~h}$ in 5\% FBS M199 supplemented with heparin and pen/strep. Then cells were stimulated by VEGF $(50 \mathrm{ng} / \mathrm{ml})$ for $18 \mathrm{~h}$ in the absence or presence of various concentrations of 6-ME. BrdU was added $6 \mathrm{~h}$ before the VEGF-induction was complete. Then indirect immunoflourescence was performed and the cells were viewed using Leica IBRE microscope. Graph indicates percentage of BrdU-incorporated cells \pm s.d. derived from four independent experiments. ${ }^{* *} p<0.0001,{ }^{* *} p=0.0013,{ }^{*} p=0.0056$.

reversible upon VEGF addition. Indeed, while $11 \%$ of the HUVECs were apoptotic, being hypodiploid in FACS analysis, after serum starvation (in 5\% FCS) (Figure 2Ai), treatment with VEGF for $18 \mathrm{~h}$ rescued almost 50\% (6\% apoptotic cells) of the cells from apoptosis (Figure 2Aii). Upon treatment of serum deprived HUVECs with
$10 \mu \mathrm{M}$ concentration of $6-\mathrm{ME}, 11.5 \%$ of HUVECs were apoptotic (Figure 2Aiii) showing no difference to the DMSO control (11\% in Figure 2Ai). Finally, treatment of serum starved HUVECs with $10 \mu \mathrm{M}$ of 6-ME did not affect the VEGF-induced survival of endothelial cells (4.1\% apoptotic cells in Figure 2Aiv). The above observations were further confirmed using Annexin/PI-apoptosis assay (Additional file1: Figure S2). These results strongly suggested that 6-ME had no effect on the survival cascades of VEGF.

6-methoxyequol does not inhibit migration of endothelial cells and tube formation in vitro

Next, we investigated the possibility that 6-ME could inhibit other processes of angiogenesis. Indeed, angiogenesis is a complex process requiring the coordinated, sequential involvement of a number of cellular events. Formation of new capillaries begins with a localized breakdown of the basement membrane of the parent vessel, followed by migration of endothelial cells for invasion of the surrounding matrix. There, a cell-matrix mediated outgrowth of an endothelial tip cell is followed by stalk cell proliferation and eventually by tube formation with an encased lumen sealed by tight cell-cell junctions. The endothelial cell migration assay and the in vitro angiogenesis assay on Matrigel recapitulate reasonably well these early events of angiogenesis. 6-ME, at $10 \mu \mathrm{M}$ concentration, did not influence the VEGFinduced migration of endothelial cells in wounded confluent monolayers of HUVECs (Figure 2B). Similarly, 6-ME, even at $50 \mu \mathrm{M}$ concentration, did not perturb capillarylike tube formation of HUVECs plated on Matrigel (Figure 2C) or the structure of the cytoskeleton (Additional file1: Figure S3). Thus, 6-ME appears to affect only endothelial cell proliferation leaving unaffected other angiogenic responses of endothelial cells.

\section{6-methoxyequol inhibits activation of the MEK1/2-ERK1/2 pathway by VEGF}

Having established that 6-ME inhibits only endothelial cell proliferation without affecting survival, migration and tube formation, we sought mechanistic confirmation of these findings. Indeed, 6-ME did not affect VEGFinduced phosphorylation of AKT (Figure 2D, upper panel), one of the key cascades that confer endothelial cell survival [11]. Likewise, 6-ME did not affect VEGFinduced phosphorylation of p38 MAPK (Figure 2D, lower panel), a signaling cascade that mediates the induction of endothelial cell migration by VEGF [12]. These results, together with the fact that 6-ME does not inhibit PLC- $\gamma$ activation, as VEGF-induced calcium release in not affected (Additional file1: Figure 4), exclude the kinase activity of VEGFR2/KDR of being the target of 6-ME. In confirmation, 6-ME clearly inhibited, 


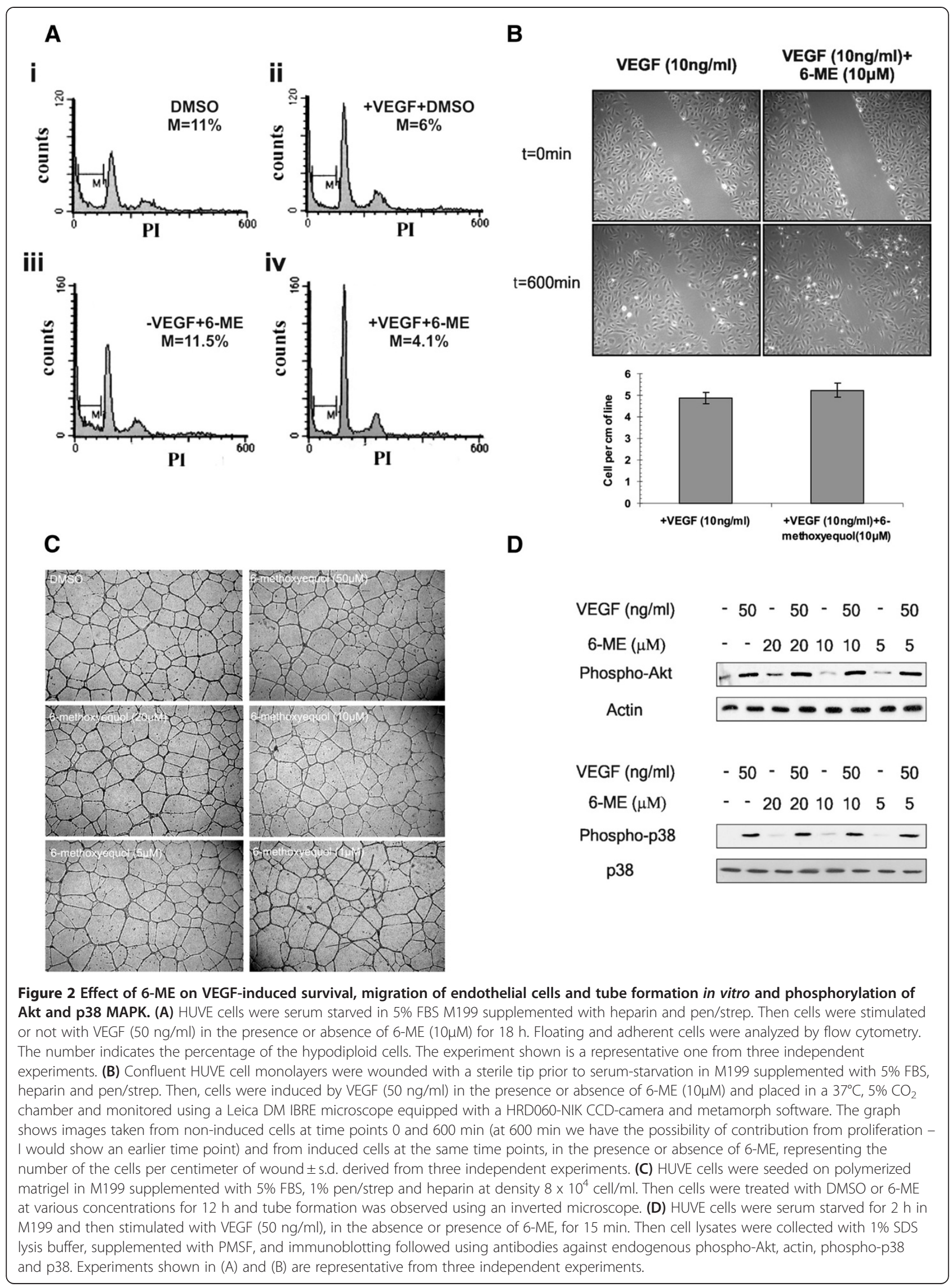


at $10 \mu \mathrm{M}$ concentration, the phosphorylation of MEK $1 / 2$ (Figure 3A) and its downstream target ERK1/2 (Figure 3B), components of the mitotic MAPK pathway that VEGF triggers via PLC- $\gamma$ activation. Several growth factors activate the ERK1/2 MAPK pathway in a Ras-dependent manner [13,14]. Indeed, 6-ME inhibited also FGF2induced phosphorylation of ERK1/2 fully compatible with the fact that 6-ME inhibited also FGF2-induced proliferation of BBCE cells (Figure 1A). To fully confirm inhibition of the ERK1/2 cascade by $6-\mathrm{ME}$, we sought additional evidence by investigating the transcriptional activation of DUSP1 and DUSP5 genes that are regulated by VEGF via the ERK1/2 pathway [15-18]. DUSP1 and DUSP5 are dual-specificity phosphatases that dephosphorylate ERK1/2 and p38 MAPK, being part of an auto-regulatory circuit [7]. Indeed, 6-ME clearly inhibited the induction of DUSP1 and DUSP5 mRNA levels by VEGF (Figure 3D) leaving no doubt that it inhibits VEGF-induced ERK1/2 activation.

\section{6-methoxyequol inhibits xenograft tumor growth only when administered directly to the tumors}

Next, we undertook the task of testing the compound in mouse xenograft tumor models. For this purpose, the synthesis of sufficient quantities of 6-ME was assured using acylation of 4-methoxyresorcinol with 4-hydroxyphenylacetic, followed by treatment of the resulting deoxybenzoin with $N, N$-dimethylformamid to yield glycitein, which was hydrogenated to 6-methoxyequol in high yield and purity (Additional file1).

We used a murine tumor xenograft model utilizing A431 cells, a human epidermoid carcinoma cell line that produces VEGF [19]. Since in previous studies we and others have found that isoflavonoids may exhibit low bioavailability [20,21], we decided to carry out two sets of experiments. In one set, 6-ME was administered orally in olive oil suspension whereas in the other, the compound was injected directly in the vicinity of the xenograft tumor.

6-ME administered orally in this model was devoid of any effect. The experimental and control tumors did not show any difference in their average volumes (Figure 4A) even though some of the experimental tumors were clearly smaller in volume compared to the control tumors (data not shown). We postulated that low bioavailability is the reason for the lack of effect. Indeed, estimation of the free, conjugated and total amounts of 6-ME in the plasma of the mice revealed that
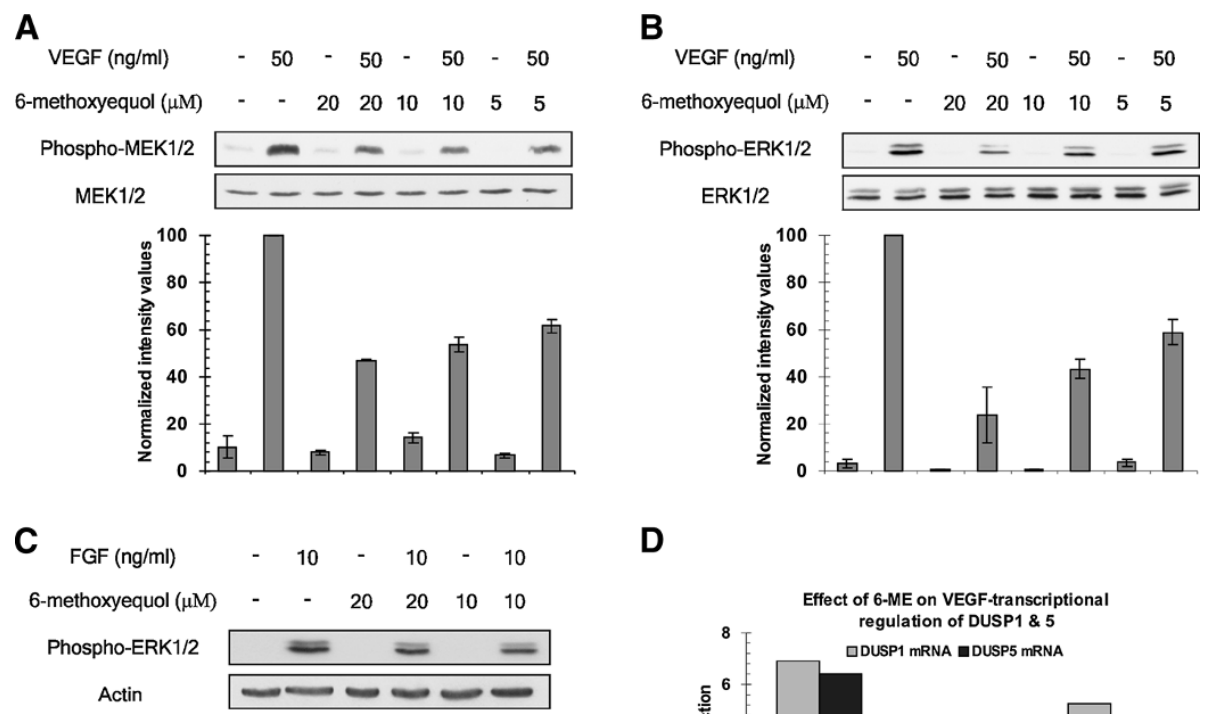

D

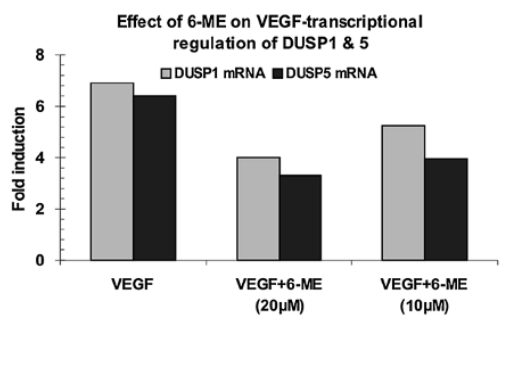

Figure 3 Effect of 6-ME on VEGF-induced phosphorylation of MEK1/2 and ERK1/2 and transcription of DUSP1 and DUSP5. HUVE cells were serum starved for $2 \mathrm{~h}$ in M199 and then stimulated with VEGF $(50 \mathrm{ng} / \mathrm{ml})$ (A \& B) or FGF $(2.5 \mathrm{ng} / \mathrm{ml})$ (C), in the absence or presence of 6 ME, for $15 \mathrm{~min}$. Then cell lysates were collected with 1\% SDS lysis buffer supplemented with PMSF and immunoblotting followed using antibodies against endogenous phospho-MEK1/2, MEK1/2, phospho-ERK1/2, ERK1/2 and actin. Graphs show normalized intensity values \pm s.d. derived from three independent experiments. (D) HUVE cells were stimulated by VEGF ( $50 \mathrm{ng} / \mathrm{ml})$ in the absence or presence of 6-ME (20, 10 $4 M$ ) for 30 min. Then, total RNA was isolated and qRT-PCR experiments followed using primers for DUSP1 and DUSP5. 
A

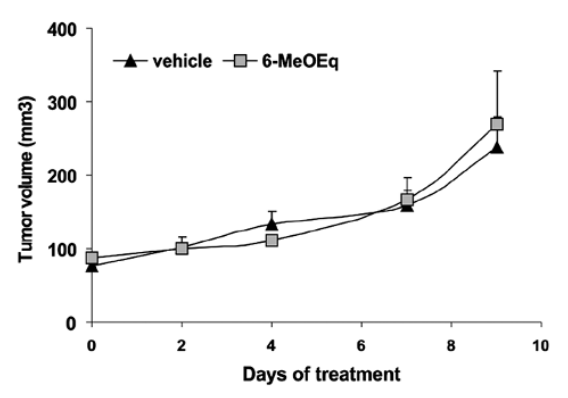

B

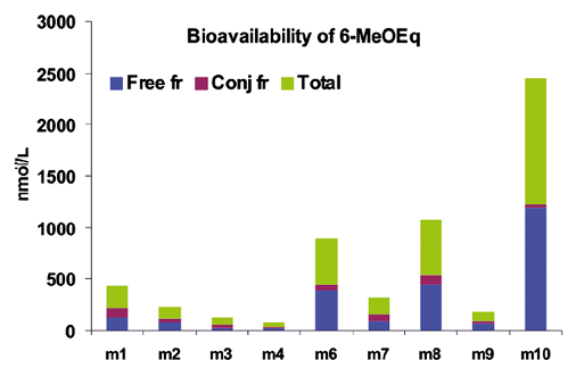

C

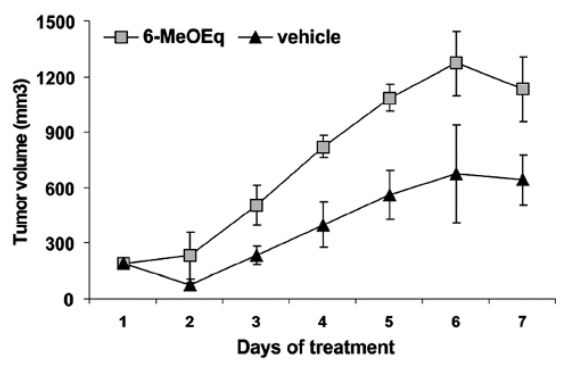

D

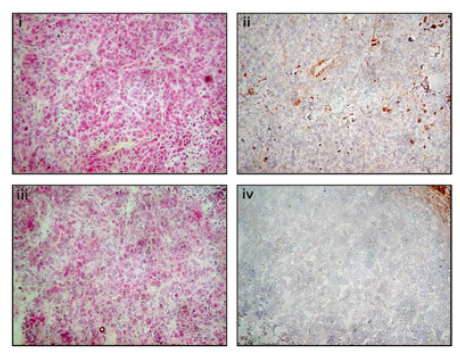

Figure 4 In vivo experiments. Female immunodeficient mice were inoculated subcutaneously in the right flank with $10^{7} \mathrm{~A}-431$ cells in a volume of $50 \mu \mathrm{l}$. When tumors reached a volume of $100 \mathrm{~mm}^{3}$ (A \& B) or $170 \mathrm{~mm}^{3}$ (C \& D), animals were randomly assigned to 2 different experimental groups and oral or peri-tumor treatment with 6-ME (5 $\mu \mathrm{g} /$ day/mice) or vehicle began, according to Materials and Methods. (A) Graph shows the tumor volume $\left(\mathrm{mm}^{3}\right) \pm$ s.d. derived from 9 animals. (B) Free fraction (Free fr), conjugated fraction (Conj fr) and total amount of 6-ME in the plasma of 9 animals ( $\mathrm{m} 1-\mathrm{m} 9)$ (C) Graph shows the tumor volume $\left(\mathrm{mm}^{3}\right) \pm$ s.d. derived from 9 animals in the case of 6-ME treated mice and 4 animals treated with vehicle, since the increased tumor volume and ulceration started from day 6 caused the death of 5 animals in this group. (D) The effect of $5 \mathrm{ug} /$ day 6-ME (panels iii-iv) on tumor angiogenesis at day 10 was compared to vehicle treated group (panels i-ii). Representative pictures of tumor sections stained with hematoxylin and eosin (i,iii) and with the antibody specific for B-FN (ii,iv).

the maximum concentration achieved was $1.23 \mu \mathrm{M}$ (Figure 4B), a value below the in vitro IC50 of the compound (around $5-10 \mu \mathrm{M}$ ).

Injecting directly the A-431 tumors with 6-ME $(5 \mu \mathrm{g} /$ day/mice) reduced the growth of tumors compared to the control group treated with vehicle. Tumors in 6-methoxyequol treated mice were significantly smaller (approximately $50 \%, \mathrm{P}<0.01$ vs. vehicle group at day 6 and 8 ) than in control mice beginning from day 2 (Figure $4 \mathrm{C}$ ). B-fibronectin (B-FN), the fibronectin (FN) isoform containing extradomain B (ED-B) accumulates around neovascular structures in aggressive tumors and other tissues undergoing angiogenesis and remodeling [22]. The monoclonal anti-ED-B antibody against the ED-B domain in fibronectin [23] indicated the presence of tumor vasculature in tumors of the control group, which was absent in 6-methoxyequol treated tumors (Figure 4D). Regarding the survival, at day 8 mice survival was $78 \%$ in the 6-methoxyequol group and $44 \%$ in the vehicle group.

\section{Discussion}

In previous studies, we have demonstrated that the isoflavonoid genistein is an angiogenesis inhibitor [8]. In the present study, we have screened a number of hitherto untested isoflavonoids using inhibition of EC proliferation as an indicator of possible anti-angiogenic activity. Only, 6-ME inhibited EC proliferation with an IC50 comparable to that of genistein or the flavonoid Luteolin (around $5 \mu \mathrm{M}$ ). Interestingly, 6-ME inhibited both VEGF- and FGF2-induced proliferation of endothelial cells, whereas it had no effect on the serum-induced proliferation of four cancer cell lines. Apparently, 6-ME exhibits certain selectivity towards inhibition of EC proliferation. 6-ME is an isoflavan metabolite that has been identified in human urine following soy or red clover supplementation $[20,21,24,25]$. However, only trace amounts of 6-ME are excreted in human urine. 6-ME originates from glycitein; the amount of the original substance is low in soy compared to daidzein and genistein, that may explain the low amounts of the metabolite [24].

Though 6-ME inhibited both VEGF- and FGF2-induced proliferation of ECs, we decided to study the effects of 6-ME only on VEGF-dependent EC responses, because VEGF is the most important mediator of tumor angiogenesis. Indeed, cancer cells over-express VEGF either following hypoxia or as a consequence of the genetic changes of cancer such as mutations of oncogenes and tumor suppressor genes [26]. In fact, endothelial cells adjacent to the tumor vessels over-express VEGFR-1 and -2 [27] establishing an angiogenic loop. 
To discriminate whether the decreased number of cells in the proliferation assay derived from a truly cytostatic effect (cell cycle inhibition) of 6-ME or was the result of cytotoxicity/apoptosis, we further investigated the effect of the compound on the VEGF-induced survival of endothelial cells. 6-ME, administered alone to endothelial cell cultures did not increase the percentage of apoptotic cells compared to solvent-treated cultures. Moreover, 6-ME administered together with VEGF did not have any influence on the VEGF-induced rescue of apoptosis. This result, in other words, indicated that 6-ME did not inhibit the EC survival signaling cascades emanating from the active VEGF/VEGFR2 complex. In confirmation, 6-ME did not inhibit VEGF-induced phosphorylation of AKT, an important component of the PI3K signaling pathway, the main anti-apoptotic cascade in most cells.

Having established that 6-ME inhibits endothelial cell proliferation, we investigated whether 6-ME could inhibit other angiogenic responses of endothelial cells. Indeed, angiogenesis is a complex process that involves many partial steps such as production of proteolytic enzymes that degrade the basement membrane, migration, proliferation, tube formation, generation of basement membrane and recruitment of mural cells [26]. Several of these processes including tube formation can be reconstituted in vitro using 3D cultures on Matrigel, a basement membrane matrix from Engelbreth-HolmSwarm mouse tumors [28]. Indeed, human umbilical vein endothelial cells form capillary-like structures on Matrigel substrates. 6-ME, even at high doses, did not exhibit any effect on the Matrigel assay. Migration is a critical angiogenic response of ECs allowing them to reach the membrane breach for invasion to the extracellular space. VEGF is a prime regulator of EC migration. VEGF-induced phosphorylation of Tyr1214 of VEGFR2 activates SAPK2/p38 [12] leading to VEGF-induced actin reorganization and migration of ECs via phosphorylation of heat-shock protein-27 (HSP27) [29] and LIM-kinase 1 (LIMK1) [6]. 6-ME did not exhibit any inhibitory effect on VEGF-induced migration of ECs and did not inhibit phosphorylation of p38 by the VEGF/VEGFR2 complex.

It appeared, therefore, that the main target of $6-\mathrm{ME}$ was EC proliferation. Interestingly, 6-ME inhibited both VEGF- and FGF2-induced EC proliferation. In humans, upon VEGF-A binding, phosphorylation of VEGFR2 on Tyr1175 leads to recruitment of PLC $\gamma$, which in turn, via activation of $\mathrm{PKC}$, phosphorylates MEK1/2 and eventually mitogen-activated protein kinase (MAPK)/ extracellular-signal-regulated kinase-1/2 (ERK1/2) leading to proliferation of ECs [5]. Such activation of MAPKs by VEGF is different from classic Ras-Raf-MEK-MAPK pathway, which is used by most receptor tyrosine kinases including FGF2 $[13,14]$. Nevertheless, it has been shown that PKC-dependent activation of MEK1/2 requires a
Ras-Raf complex formation [30]. This PKC/Ras-Raf functional interaction is not so well understood and might include other hitherto unidentified components. PKC and Ras-Raf are the points where the VEGF and FGF2 cascades arrive just before the first downstream common effector, MEK1/2, as far as activation of MAPK is concerned. The finding that 6-ME inhibits both the VEGF and FGF2-induced EC proliferation as well as MEK1/2 phosphorylation suggests that the PKC/Ras-Raf interaction is the only point where 6-ME could target both pathways with one activity. Otherwise, 6-ME would need two activities targeting two different components upstream to MEK1/2, one for each pathway. This is a point that requires future attention.

Thus, inhibition of MEK $1 / 2$ and consequently ERK1/2 phophorylation was the sole cardinal effect of 6 -ME on the signaling cascade of VEGF in HUVECs; activation of AKT and P38 were unaffected. This mechanism is strikingly different compared to the effects of the flavonoid luteolin on VEGF signaling in HUVECs [10]. Luteolin, inhibited the PI3K/AKT pathway abolishing downstream survival signals, but also enhanced the pro-apoptotic MKK3/MKK6/p38 pathway of VEGF eliciting a strong apoptotic effect in ECs. Regarding the anti-mitotic activity, luteolin inhibited VEGF-induced phosphorylation of p70 S6K, a downstream effector of PI3K responsible for G1 progression. Surprisingly, luteolin did not affect VEGF-induced phosphorylation of ERK1/2 MAP kinases. Thus, two representatives (luteolin and 6-ME) of closely related isomeric compound classes (flavonoids and Isoflavonoids) exhibited entirely different molecular targets concerning the VEGF-dependent signaling cascades in HUVECs. Perhaps, the fact that these compounds are competitive inhibitors of ATP binding [31] allows them to target a variety of tyrosine and serine kinases $[31,32]$.

6-ME was eventually tested in animal models. For this purpose, we used a murine tumor xenograft model utilizing A-431 cells, a human epidermoid carcinoma cell line that produces VEGF [19]. 6-ME administered orally in this model was devoid of any effect. The experimental and control tumors did not show any difference in their average volumes). We postulated that low bioavailability is the reason for the lack of effect. Indeed, estimation of the free, conjugated and total amounts of 6-ME in the plasma of the mice revealed that the maximum concentration achieved was $1.23 \mu \mathrm{M}$, a value below the in vitro IC50 of the compound (around 5-10 $\mu \mathrm{M}$ ). Several factors contribute to the bioavailability including absorption, distribution, metabolism and elimination. There are no extensive studies on these issues concerning isoflavonoids. However, the studies so far $[20,21]$ anticipate that isoflavones are rather poorly bioavailable. In a study in human adults, consumption of $50 \mathrm{mg}$ of isoflavones per day yielded plasma concentrations ranging 
from 0.2-3.2 $\mu \mathrm{mol} / \mathrm{L}$. Indeed, following consumption of food rich in soy or red clover only traces of 6-ME were detected in soy human urine [24]. The low biovailability excludes any significant contribution of 6-ME to the protective function of plant-based diets on cancer incidence.

However, biovailable analogs of 6-ME could be used therapeutically to target tumor angiogenesis. Alternatively, 6-ME could be loaded in nanoparticles targeted to ECs, where they could be endocytosed and eventually release their cargo. Indeed, when injected directly to the xenograft tumors, to bypass its low biovailability, 6-ME suppressed tumor vascularization resulting to a statistically significant decrease in the volumes of murine A-431 xenograft tumors. Thus, 6-ME acquires the potential to be developed into a therapeutic anti-cancer agent. In this capacity, 6-ME or 6-ME analogs have two very important and unique properties. 6-ME inhibits only VEGF-induced MEK1/2 activation inhibiting exclusively EC proliferation without influencing VEGF-induced survival. Thus, one can anticipate that it targets only dividing ECs in the vicinity of tumors, without affecting the survival of the quiescent normal endothelium. Moreover, it inhibits also FGF2, which an alternative angiogenic factor expressed when ECs develop resistance (Angiogenic Redundancy) [33] against current anti-VEGF treatments [34]. This is a very important issue in the anti-VEGF treatments.

In conclusion, 6-ME, a natural isoflavone found also in humans, inhibits VEGF- and FGF2-induced proliferation of ECs. The molecular target of 6-ME is upstream of MEK1/2 inhibiting phosphorylation of MEK1/2 and ERK1/2 kinases that are important components of the mitogenic MAPK pathway. 6-ME does not affect the PI3K/AKt pathway, thereby not affecting VEGF-dependent survival of ECs. Oral administration in mice fails to achieve sufficient plasma concentrations to inhibit neovascularization and growth of xenograft tumors in mice. However, direct injections of 6-ME to the xenograft tumors, to bypass its low biovailability, suppress tumor vascularization resulting to a statistically significant decrease in the volumes of murine A-431 xenograft tumors. Concomitant inhibition of VEGF- and FGF2-induced EC proliferation and targeting only dividing ECs without affecting the survival of ECs are two properties rendering 6-ME as an attractive molecule for the development of a novel anti-angiogenic intervention in cancer treatment.

\section{Additional file}

Additional file 1 Scheme 1: Synthesis of 6-Methoxyequol. Additional file 1: Figure1 Effect of 6-ME on primary human fibroblast proliferation. Additional file 1: Figure2 Effect of 6-ME on VEGF-induced survival of endothelial cells. Additional file 1: Figure 3 Effect of 6-ME on the structure of actin filaments and microtubules. (DOC $8891 \mathrm{~kb}$ )

\section{Competing interests}

The authors declare that they have no competing interests.

\section{Acknowledgements}

The skillful technical assistance of Lambrini Kyrkou is gratefully acknowledged.

\section{Grant support}

Work at the University of loannina, FORTH/BRI and Folkhälsan Research Centre was supported by a research grant from the European Commission Contract No. QLRT-2000-00266 (PHYTOPREVENT).

\section{Author details}

${ }^{1}$ Department of Engineering Informatics and Telecommunications, University of Western Macedonia, 50100, Kozani, Greece. ${ }^{2}$ Department of Biomedical Research, Foundation of Research and Technology-Hellas, Institute of Molecular Biology \& Biotechnology, University Campus, 45110, loannina, Greece. ${ }^{3}$ School of Chemistry, University of St Andrews, St Andrews, Fife, KY16 9ST, Scotland, UK. ${ }^{4}$ Department of Molecular Biology, University of Siena, Via Aldo Moro 2, 53100, Siena, Italy. ${ }^{5}$ Folkhälsan Research Centre, and Department of Clinical Chemistry, Biomedicum, University of Helsinki, P.O. Box 63FIN-00014, Helsinki, Finland. 'Laboratory of Biological Chemistry, Medical School, University of Ioannina, 45110, Ioannina, Greece.

Received: 15 December 2011 Accepted: 14 May 2012

Published: 14 May 2012

\section{References}

1. Grothey A, Galanis E: Targeting angiogenesis: progress with anti-VEGF treatment with large molecules. Nat Rev Clin Oncol 2009, 6(9):507-18.

2. Ferrara N, Gerber HP, LeCouter J: The biology of VEGF and its receptors. Nat Med 2003, 9(6):669-76.

3. Tozer GM, et al: Blood vessel maturation and response to vasculardisrupting therapy in single vascular endothelial growth factor-A isoform-producing tumors. Cancer Res 2008, 68(7):2301-11.

4. Dvorak HF: Vascular permeability factor/vascular endothelial growth factor: a critical cytokine in tumor angiogenesis and a potential target for diagnosis and therapy. J Clin Oncol 2002, 20(21):4368-80.

5. Takahashi T, et al: A single autophosphorylation site on KDR/Flk-1 is essential for VEGF-A-dependent activation of PLC-gamma and DNA synthesis in vascular endothelial cells. EMBO J 2001, 20(11):2768-78.

6. Kobayashi M, et al: MAPKAPK-2-mediated LIM-kinase activation is critical for VEGF-induced actin remodeling and cell migration. EMBO J 2006, 25(4):713-26.

7. Bellou $\mathrm{S}$, et al: VEGF Auto-regulates its Proliferative and Migratory ERK $1 / 2$ and p38 Cascades by Enhancing the Expression of DUSP1 and DUSP5 Phosphatases in Endothelial Cells. Am J Physiol Cell Physiol 2009, 297(6):1477-1489.

8. Fotsis T, et al: Genistein, a dietary-derived inhibitor of in vitro angiogenesis. Proc Natl Acad Sci U S A 1993, 90(7):2690-4.

9. Fotsis T, et al: Flavonoids, dietary-derived inhibitors of cell proliferation and in vitro angiogenesis. Cancer Res 1997, 57(14):2916-21.

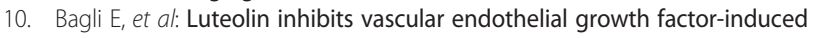
angiogenesis; inhibition of endothelial cell survival and proliferation by targeting phosphatidylinositol 3'-kinase activity. Cancer Res 2004, 64(21):7936-46.

11. Gerber HP, et al: Vascular endothelial growth factor regulates endothelial cell survival through the phosphatidylinositol 3'-kinase/Akt signal transduction pathway. Requirement for Flk-1/KDR activation. J Biol Chem 1998, 273(46):30336-43.

12. Lamalice $L$, et al: Phosphorylation of tyrosine 1214 on VEGFR2 is required for VEGF-induced activation of Cdc42 upstream of SAPK2/p38. Oncogene 2004, 23(2):434-45

13. Wang JK, et al: Broadly expressed SNT-like proteins link FGF receptor stimulation to activators of Ras. Oncogene 1996, 13(4):721-9.

14. Kouhara $\mathrm{H}$, et al: A lipid-anchored Grb2-binding protein that links FGF-receptor activation to the Ras/MAPK signaling pathway. Cell 1997, 89(5):693-702.

15. Gomez AR, et al: Conserved cross-interactions in Drosophila and Xenopus between Ras/MAPK signaling and the dual-specificity phosphatase MKP3. Dev Dyn 2005, 232(3):695-708. 
16. Kucharska A, et al: Regulation of the inducible nuclear dual-specificity phosphatase DUSP5 by ERK MAPK. Cell Signal 2009, 21(12):1794-1805.

17. Hu JH, et al: Feedback control of MKP-1 expression by p38. Cell Signal 2007, 19(2):393-400.

18. McMullen ME, et al: Activation of p38 has opposing effects on the proliferation and migration of endothelial cells. J Biol Chem 2005, 280(22):20995-1003.

19. Myoken $Y$, et al: Vascular endothelial cell growth factor (VEGF) produced by A-431 human epidermoid carcinoma cells and identification of VEGF membrane binding sites. Proc Natl Acad Sci U S A 1991, 88(13):5819-23.

20. Heinonen SM, et al: Studies of the in vitro intestinal metabolism of isoflavones aid in the identification of their urinary metabolites. J Agric Food Chem 2004, 52(9):2640-6.

21. Xu X, et al: Bioavailability of soybean isoflavones depends upon gut microflora in women. J Nutr 1995, 125(9):2307-15.

22. Borsi $L$, et al: Selective targeted delivery of TNFalpha to tumor blood vessels. Blood 2003, 102(13):4384-92.

23. Pini A, et al: Design and use of a phage display library. Human antibodies with subnanomolar affinity against a marker of angiogenesis eluted from a two-dimensional gel. J Biol Chem 1998, 273(34):21769-76.

24. Heinonen SM, et al: Metabolism of the soy isoflavones daidzein, genistein and glycitein in human subjects. Identification of new metabolites having an intact isoflavonoid skeleton. J Steroid Biochem Mol Biol 2003, 87(4-5):285-99.

25. Heinonen SM, Wahala K, Adlercreutz H: Identification of urinary metabolites of the red clover isoflavones formononetin and biochanin A in human subjects. J Agric Food Chem 2004, 52(22):6802-9.

26. Carmeliet $P$, Jain RK: Molecular mechanisms and clinical applications of angiogenesis. Nature, 473(7347):298-307.

27. Ferrara N, Davis-Smyth T: The biology of vascular endothelial growth factor. Endocr Rev 1997, 18(1):4-25.

28. Kleinman HK, Martin GR: Matrigel: basement membrane matrix with biological activity. Semin Cancer Biol 2005, 15(5):378-86.

29. Rousseau $\mathrm{S}$, et al: p38 MAP kinase activation by vascular endothelial growth factor mediates actin reorganization and cell migration in human endothelial cells. Oncogene 1997, 15(18):2169-77.

30. Doanes AM, et al: VEGF stimulates MAPK through a pathway that is unique for receptor tyrosine kinases. Biochem Biophys Res Commun 1999, 255(2):545-8.

31. Graziani Y, Erikson E, Erikson RL: The effect of quercetin on the phosphorylation activity of the Rous sarcoma virus transforming gene product in vitro and in vivo. Eur J Biochem 1983, 135(3):583-9.

32. Cunningham $\mathrm{BD}$, et al: Synthesis and biological evaluation of a series of flavones designed as inhibitors of protein tyrosine kinases. Anticancer Drug Des 1992, 7(5):365-84.

33. Ribatti D: Novel angiogenesis inhibitors: addressing the issue of redundancy in the angiogenic signaling pathway. Cancer Treat Rev 2011 37(5):344-52.

34. Grepin R, Pages G: Molecular mechanisms of resistance to tumour antiangiogenic strategies. J Oncol 2010, 2010:835680.

doi:10.1186/1476-4598-11-35

Cite this article as: Bellou et al:: The isoflavone metabolite 6methoxyequol inhibits angiogenesis and suppresses tumor growth. Molecular Cancer 2012 11:35.

\section{Submit your next manuscript to BioMed Central and take full advantage of:}

- Convenient online submission

- Thorough peer review

- No space constraints or color figure charges

- Immediate publication on acceptance

- Inclusion in PubMed, CAS, Scopus and Google Scholar

- Research which is freely available for redistribution 